\title{
Coupling effects of beam-beam interaction and longitudinal impedance
}

\author{
Chuntao Lin $\odot,{ }^{1,2}$ Kazuhito Ohmi $\oplus^{3, *}$ and Yuan Zhang $\oplus^{1,2, \uparrow}$ \\ ${ }^{1}$ Key Laboratory of Particle Acceleration Physics and Technology, Institute of High Energy Physics, \\ Chinese Academy of Sciences, Beijing 100049, China \\ ${ }^{2}$ University of Chinese Academy of Sciences, Beijing 100049, China \\ ${ }^{3}$ KEK, Tsukuba, Ibaraki 305-0801, Japan
}

(Received 25 September 2021; accepted 10 January 2022; published 24 January 2022)

\begin{abstract}
Recent studies have shown a novel coherent head-tail instability induced by beam-beam interaction with a large Piwinski angle. The instability has become an important issue during the designs of CEPC and FCC-ee. Simulations have further revealed that the longitudinal impedance has a strong impact on the beam stability, squeezing the horizontal stable tune area seriously. The cross-wake force has been introduced to represent beam-beam interaction. A mode coupling theory based on the localized wake (impedance) force has been developed to explain the instability. However, the theory did not consider the effects of longitudinal impedance. In this paper, we develop a new transverse mode coupling analysis method that could be used to study the beam-beam instability with and without longitudinal impedance. The result shows that the distortion of longitudinal phase space trajectory and the incoherent synchrotron tune shift induced by longitudinal impedance would reduce the stable tune area.
\end{abstract}

DOI: 10.1103/PhysRevAccelBeams.25.011001

\section{INTRODUCTION}

Beam-beam interaction with a crossing angle has been studied for many years. It has been noticed that increasing the Piwinski angle $\left(\theta_{P}=\frac{\sigma_{z}}{\sigma_{x}} \theta_{c}\right)$ may help to achieve a higher luminosity [1]. However, only with a large Piwinski angle, there exists very strong transverse nonlinear resonance [2] coming from beam-beam interaction. The crab waist collision scheme was proposed to suppress the resonance by placing sextupoles before and after interaction point (IP) with proper phase advances, which also helps increase the beam-beam parameter [3]. This crab waist scheme has been successfully tested to achieve a high luminosity [4]. Several future colliders would adopt this kind of collision scheme. With a large Piwinski angle, the horizontal beam-beam parameter is normally very low $\left(\xi_{x}<0.01\right)$. Usually, it is believed that the horizontal oscillation of colliding bunch would be very stable. However, during the study of FCC-ee, the simulations $[5,6]$ showed that there exists a coherent head-tail instability (X-Z instability) in collision with a large Piwinski angle. The instability is observed when the strong-strong model is used. The cross-wake force induced by beam-

*Corresponding author.ohmi@post.kek.jp

Published by the American Physical Society under the terms of the Creative Commons Attribution 4.0 International license. Further distribution of this work must maintain attribution to the author(s) and the published article's title, journal citation, and DOI. beam interaction has been introduced to successfully explain this newfound instability $[7,8]$.

The stability of horizontal motion is sensitive to the longitudinal dynamics. The longitudinal impedance would modify the beam distribution, distort the longitudinal phase space trajectory, and produce incoherent synchrotron tune shift. Strong-strong simulation [9] showed that the stable tune area would be shifted, and the width would be squeezed when the longitudinal impedance is included in the simulation. It is interesting to study how the longitudinal impedance influences the $\mathrm{X}-\mathrm{Z}$ instability.

The ordinary transverse mode coupling instability (TMCI) theory [10] is derived as a perturbed Vlasov equation. In this theory, the transverse impedance, a perturbation source, represents the averaged wake force around the circumference of the ring. If one employs this ordinary approach to study beam-beam interaction, the localized property of cross-wake force would not be taken into account, which would not exhibit instability [8]. Moreover, since the TMCI is based on the solution of Sacherer's integral equation, only a few analytic solutions are known for some specific beam distributions so far. Some transverse mode coupling analytical methods have been developed to treat the localized wake force $[7,8,11]$. However, the distortion of longitudinal phase space trajectory and the incoherent synchrotron tune shift were not considered in these papers. Other methods that could consider these facts, such as the discretization method proposed by Oide [12] and the Laguerre polynomial expansion method proposed by Cai [13], just apply to longitudinal instability problems. In short, the above- 
mentioned methods cannot be applied directly to analyze the horizontal beam-beam instability with longitudinal impedance.

In this paper, we will develop a new transverse mode coupling analysis method where the effects of longitudinal phase space trajectory distortion and incoherent synchrotron tune shift induced by longitudinal impedance could be considered.

This paper is organized as follows. The longitudinal motion with wakefield is discussed in Sec. II. In Sec. III, we introduce our transverse mode coupling analysis method; and then, in Sec. IV, we apply our approach to cases with and without longitudinal impedance. Some discussions and conclusions are given in the end.

\section{LONGITUDINAL MOTION WITH WAKEFIELD}

\section{A. Hamiltonian with potential-well distortion}

We use $s=z+v_{0} t$ with $s$ the longitudinal Serret-Frenet coordinate, representing the arc length measured along the closed orbit from an initial point, $v_{0} \approx c$ the synchronous velocity and $t$ clock time. $z$ is the longitudinal distance from the synchronous particle and $z>0$ is the bunch head. In the following, we will use $s$ as the timelike variable and $z$ as the longitudinal coordinate.

As the particle moves along the beamline, the head of the bunch will act as a source of an electromagnetic field that kicks the tail. In one revolution, the relative longitudinal momentum kick $\Delta \delta(z)$ received by a particle at $z$ can be expressed by a wake function [10],

$$
\Delta \delta(z)=-\frac{N_{0} r_{e}}{\gamma} \int_{-\infty}^{\infty} W_{z}\left(z-z^{\prime}\right) \rho\left(z^{\prime}\right) d z^{\prime}
$$

$W_{z}(z)$ is the ordinary longitudinal wake function with the property $W_{z}(z)=0(z>0)$. $N_{0}$ represents the single bunch population, $r_{e}$ is the classical radius of the electron, $\gamma$ is the relativistic factor and $\rho(z)$ is normalized line density.

Without the longitudinal wakefield $W_{z}(z)$, the Hamiltonian of a single particle is described by a simple harmonic oscillator

$H_{0}=-\frac{\eta_{p}}{2} \delta^{2}-\frac{\mu_{z}^{2}}{2 \eta_{p} L^{2}} z^{2}, \quad \sigma_{z}=\frac{\eta_{p} L}{\mu_{z}} \sigma_{\delta} \equiv \beta_{z} \sigma_{\delta}$.

$L$ represents the circumference of the ring, $\nu_{s}$ is the synchrotron tune, $\mu_{z}=2 \pi \nu_{s}, \eta_{p}$ is the slippage factor, $\sigma_{z}$ is the rms bunch length, and $\sigma_{\delta}$ is relative rms energy spread, respectively. Including the longitudinal wakefield, the Hamiltonian of the particle then reads,

$$
\begin{aligned}
-H= & \frac{\eta_{p}}{2} \delta^{2}+\frac{\mu_{z}^{2}}{2 \eta_{p} L^{2}} z^{2}-\frac{1}{L} \frac{N_{0} r_{e}}{\gamma} \int_{0}^{z} d z^{\prime \prime} \\
& \times \int_{-\infty}^{\infty} d z^{\prime} W_{z}\left(z^{\prime \prime}-z^{\prime}\right) \rho\left(z^{\prime}\right) \\
\equiv & \frac{\eta_{p}}{2} \delta^{2}+\frac{\mu_{z}^{2}}{2 \eta_{p} L^{2}} z^{2}-\frac{1}{L} V(z),
\end{aligned}
$$

or in normalized form,

$-\frac{H}{\eta_{p} \sigma_{\delta}^{2}}=\frac{\delta^{2}}{2 \sigma_{\delta}^{2}}+\left[\frac{z^{2}}{2 \sigma_{z}^{2}}-\frac{1}{\eta_{p} L \sigma_{\delta}^{2}} V(z)\right] \equiv \frac{\delta^{2}}{2 \sigma_{\delta}^{2}}+V_{P W}(z)$,

where we define the potential-well $V_{P W}(z)$ as follows,

$$
V_{P W}(z)=\underbrace{\frac{z^{2}}{2 \sigma_{z}^{2}}}_{\text {byRF }} \underbrace{-\frac{1}{\mu_{z} \sigma_{z} \sigma_{\delta}} V(z)}_{\text {byWz }} .
$$

In the following, we will use a parallel RLC resonator model to describe the longitudinal wakefield,

$W_{z}(z)=\alpha R_{S} e^{\alpha z / c}\left(\cos \frac{\bar{\omega} z}{c}+\frac{\alpha}{\bar{\omega}} \sin \frac{\bar{\omega} z}{c}\right), \quad z<0$.

where $\alpha=\omega_{R} / 2 Q, \bar{\omega}=\sqrt{\omega_{R}^{2}-\alpha^{2}} . \omega_{R}$ is the resonant frequency. Although we use the resonator model, our analysis is applicable for any form of wakefield. The resonator parameters are chosen by fitting the momentum kick $\Delta \delta(z)$ which is obtained by CEPC impedance [14]. Here we choose $\omega_{R}=2 \pi \times 56 \times 10^{9} \mathrm{rad} / \mathrm{s}, \quad R_{s}=891898 \Omega$, $Q=10$. Figure 1 shows each potential term of Eq. (5) where $\sigma_{z}=7.35 \mathrm{~mm}, \sigma_{\delta}=1.055 \times 10^{-3}$. Some parameters [15] used in the paper are listed in Table I.

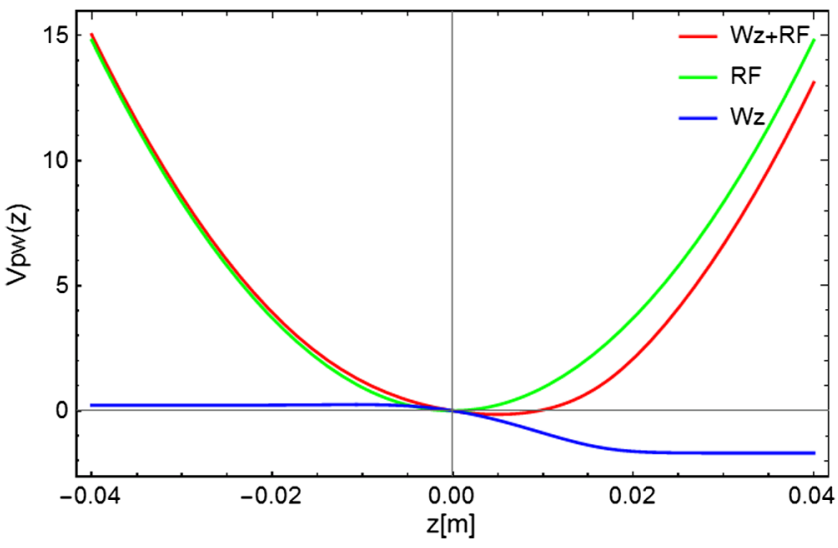

FIG. 1. Each term of the potential $V_{P W}(z)$. The green line is the potential generated by rf cavity $(r f)$, the blue is wake potential $\left(W_{z}\right)$, and the red is the total potential $(r f+W z)$, where $\sigma_{z}=7.35 \mathrm{~mm}, \sigma_{\delta}=1.055 \times 10^{-3}$. 
TABLE I. CEPC-Z half ring parameters.

\begin{tabular}{lcc}
\hline \hline Parameters & & \\
\hline Circumference & $L(\mathrm{~km})$ & 50 \\
Synchrotron tune & $\nu_{s}$ & 0.014 \\
Beta function at IP & $\beta_{x}^{*}(\mathrm{~m})$ & 0.15 \\
Beam energy & $E(\mathrm{GeV})$ & 45.5 \\
Horizontal emittance & $\epsilon_{x}(\mathrm{~nm})$ & 0.18 \\
Half crossing angle & $\theta_{c}(\mathrm{mrad})$ & 16.5 \\
rms bunch length & $\sigma_{z}(\mathrm{~mm})$ & 7.35 \\
Energy spread & $\sigma_{\delta}\left(10^{-3}\right)$ & 1.055 \\
Bunch population & $N_{0}\left(10^{10}\right)$ & 8 \\
\hline \hline
\end{tabular}

The Vlasov equation describes the evolution of the bunch distribution $\psi(z, \delta)$ under the influence of wakefield. The stationary solution [10] to the Vlasov equation must be a function of $H$,

$$
\psi(z, \delta)=\psi(H)
$$

For electron machine, due to the synchrotron radiation, the stationary distribution should have a Gaussian distribution with the rms value $\sigma_{\delta}$ in $\delta$,

$$
\psi(z, \delta)=\frac{1}{\sqrt{2 \pi} \sigma_{\delta}} \exp \left(-\frac{\delta^{2}}{2 \sigma_{\delta}^{2}}\right) \rho(z)
$$

This exponential, when combined with the Hamiltonian $H$ in Eq. (3) and stationary solution in Eq. (7), gives

$$
\psi(z, \delta) \propto \exp \left(\frac{H}{\eta_{p} \sigma_{\delta}^{2}}\right)
$$

$H$ can be regarded as a function of action $J, H=H(J)$, meaning that the stationary phase space distribution only depends on $J$. Integrating both sides of Eq. (9) over $\delta$, we finally arrive at a self-consistent equation, the Haissinski equation [16], for the linear density

$$
\begin{aligned}
\rho(z)= & \rho_{0} \exp \left[-\frac{1}{2}\left(\frac{\mu_{z} z}{\eta_{p} L \sigma_{\delta}}\right)^{2}+\frac{N_{b} r_{e}}{\eta_{p} \sigma_{\delta}^{2} L \gamma} \int_{0}^{z} d z^{\prime \prime}\right. \\
& \left.\times \int_{z^{\prime \prime}}^{\infty} d z^{\prime} \rho\left(z^{\prime}\right) W_{z}^{\prime}\left(z^{\prime \prime}-z^{\prime}\right)\right],
\end{aligned}
$$

where the constant $\rho_{0}$ is obtained by normalizing $\rho(z)$ to unity.

We use the wake function in Eq. (6) to numerically solve this integral equation. Figure 2 shows the comparison of Gaussian distribution and equilibrium Haissinski distribution. When the longitudinal wakefield is included, the bunch length is lengthened from $\sigma_{z}=7.35 \mathrm{~mm}$ to $\sigma_{z}=8.03 \mathrm{~mm}$.

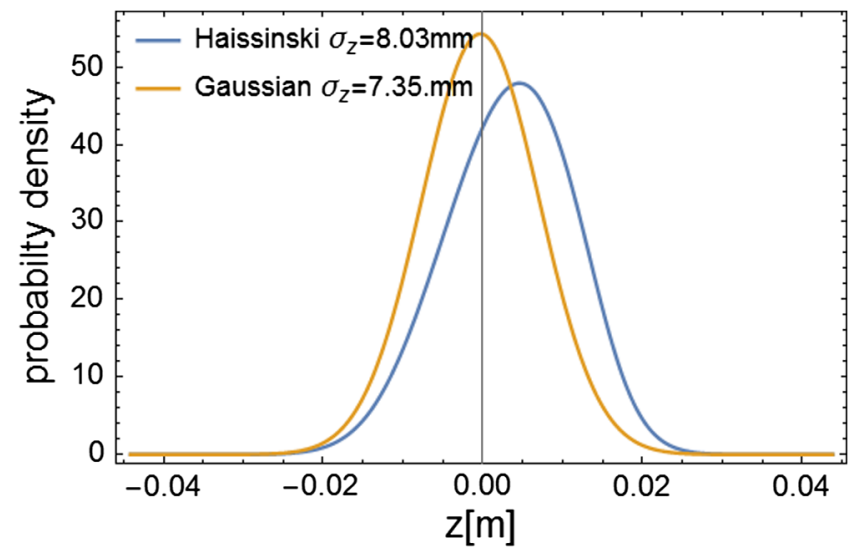

FIG. 2. Comparison of Haissinski distribution of the design population in the CEPC-Z to a Gaussian distribution.

\section{B. Action-angle variables and Synchrotron tune}

Particles move along closed $\delta-z$ orbits in longitudinal phase space. Each closed orbit corresponds to a particular action $J$ and an energy $E$ ( $E$ is the value of Hamiltonian $H$ ). The Hamiltonian of a particle is one-dimensional and timeindependent, thus, we expect to solve its motion numerically. For each specific orbit, using the Hamiltonian in Eq. (4), we could derive the action $J$ [17],

$$
\begin{aligned}
J(E) & =\frac{1}{2 \pi} \oint \delta d z \\
& =\frac{1}{\pi} \int_{z_{\min }}^{z_{\max }} \sqrt{-2 \sigma_{\delta}^{2}\left(V_{P W}(z)+\frac{E}{\eta_{p} \sigma_{\delta}^{2}}\right)} d z,
\end{aligned}
$$

where $z_{\min }, z_{\max }$ are the turning points in the longitudinal phase space as shown in Fig. 3.

After determining $J$, we now use the second type of generating function $F_{2}(z, J)$ [17] to get its conjugate coordinate $\phi$. Note that $J$ is a function of $E, J=J(E)$, thus, we have $F_{2}(z, J) \equiv \tilde{F}_{2}[z, E(J)]$. Using the properties of generating function, we have the relation,

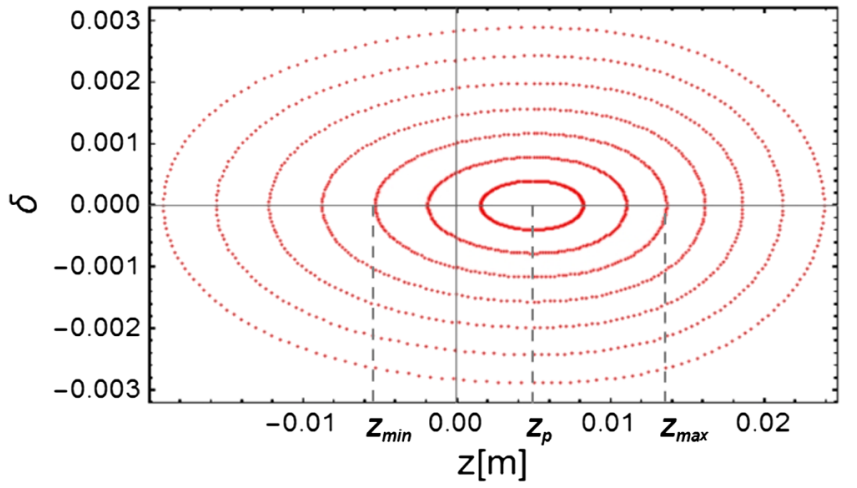

FIG. 3. Distorted particle's trajectory in longitudinal phase space. Each circular set of points corresponds to a specific value of Hamiltonian. 


$$
\delta=\partial F_{2} / \partial z
$$

Substituting $\delta$ in Eq. (4) into Eq. (12) and integrating both sides over $z$, we obtain

$\tilde{F}_{2}[z, E(J)]=\int_{z_{\min }}^{z} d z^{\prime} \sqrt{-2 \sigma_{\delta}^{2}\left(V_{P W}\left(z^{\prime}\right)+\frac{E}{\eta_{p} \sigma_{\delta}^{2}}\right)}$,

For a general potential well $V_{P W}\left(z^{\prime}\right)$, the integral may not be analytically calculated but can be numerically computed.

After the generating function $\tilde{F}_{2}[z, E(J)]$ is obtained by numerical method, we can compute the conjugate angular variable $\phi$. According to the property of generating function, we have

$$
\phi=\frac{\partial F_{2}(z, J)}{\partial J}=\frac{\partial \tilde{F}_{2}[z, E(J)]}{\partial J} .
$$

The discrete phase space points shown in Fig. 3 can be used in the numerical integration of Eq. (11). For a specific point $(z, \delta), J$ is obtained. Then, $\phi$ is easily calculated by Eq. (14). The map from $(z, \delta)$ to $(J, \phi)$ is finally determined. The techniques for sampling $(z, \delta)$ points are not unique. Here the sampling is taken as follows,

$$
z_{i}=\left\{\begin{array}{ll}
z_{p}+\left(z_{\max }-z_{p}\right) \cos \frac{i \pi}{N_{\phi}} & 0 \leq i \leq \frac{N_{\phi}}{2} \\
z_{p}+\left(z_{p}-z_{\min }\right) \cos \frac{i \pi}{N_{\phi}} & \frac{N_{\phi}}{2}<i \leq N_{\phi}
\end{array},\right.
$$

where $z_{p}$ is the zero point of potential-well satisfying $V_{P W}\left(z_{p}\right)=0$, and $N_{\phi}=100$.

We now calculate the synchrotron tune $\nu_{S}(J)$ as a function of $J$ from the Hamiltonian equation. Considering $E$ as the new Hamiltonian function, $J$ as the new momentum and its conjugate angle coordinate $\phi$ as the new position, we have Hamilton's equation of motion [17]

$$
\frac{d \phi}{d s}=\frac{d E}{d J}
$$

The synchrotron tune is, therefore, expressed as

$$
\nu_{s}(J)=\frac{L}{2 \pi} \frac{d E}{d J} .
$$

This can be calculated numerically using Eq. (11).

Another way to obtain $\nu_{s}(J)$ is that according to Eq. (4), Hamilton's equation is

$$
\frac{d z}{d s}=\frac{\partial H}{\partial \delta}=-\eta_{p} \delta
$$

After rearrangement, we have

$$
d s=-\frac{d z}{\eta_{p} \delta} .
$$

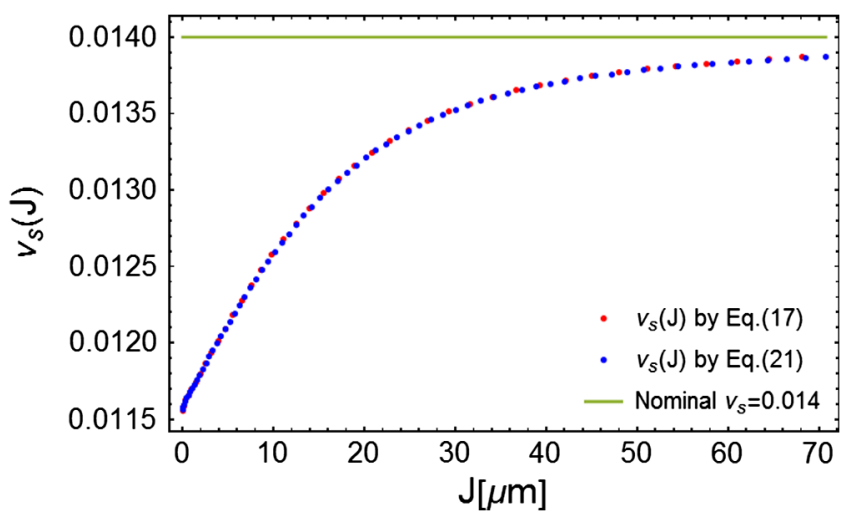

FIG. 4. Synchrotron tune $\nu_{s}(J)$ as a function of $J$ at the design population. The red and blue dots are the calculated synchrotron tune using two different methods: Eq. (17) and Eq. (21), respectively. The green line is nominal synchrotron tune $\nu_{s}=0.014$.

When a particle moves one circle in phase space, the distance of the particle traveling along the accelerator ring is

$$
\Delta s(E)=\int_{s_{1}}^{s_{2}} d s=2 \int_{z_{\min }}^{z_{\max }} \frac{1}{-\eta_{p} \delta(E)} d z
$$

Therefore, the synchrotron tune $\nu_{s}$ can be expressed as,

$$
\nu_{s}(E)=\frac{L}{\Delta s(E)} .
$$

Figure 4 shows the nominal synchrotron tune and incoherent synchrotron tune shift calculated by the abovementioned two methods. As one can see from the figure, the tune shift mostly occurs at small amplitude $\Delta \nu_{s} \approx$ 0.00225 , and it asymptotically vanishes at large amplitude.

\section{EIGENVALUE PROBLEM FOR LOCALIZED SINGLE-BEAM WAKE FORCE WITH LONGITUDINAL IMPEDANCE}

\section{A. Cross-wake force induced by beam-beam force}

Here we briefly review the definition of cross-wake force. Considering electron $\left(e^{-}\right)$and positron $\left(e^{+}\right)$bunches

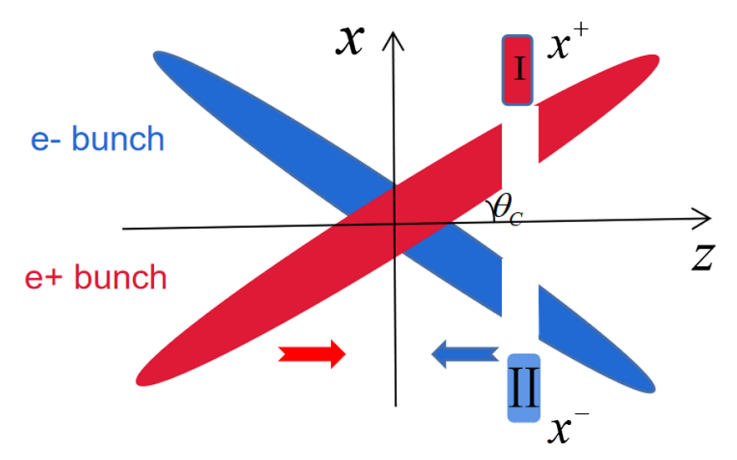

FIG. 5. Beam-beam interaction with a crossing angle in Lorentz boost frame. 
colliding with half crossing angle $\theta_{c}$ in the $x-z$ plane, the two bunches head on each other tilted horizontally by $\theta_{c}$ in the Lorentz boost frame [18] shown in Fig. 5. As the collision proceeds from the head of the bunch to the tail, the perturbed momentum kick due to horizontal betatron oscillation experienced by a $e^{\mp}$ particle at $z$ is expressed as follows [8],

$$
\begin{aligned}
\Delta p_{x}^{(\mp)}(z)= & -\int_{-\infty}^{\infty} W_{x}^{(\mp)}\left(z-z^{\prime}\right) \rho_{x}^{( \pm)}\left(z^{\prime}\right) d z^{\prime} \\
& +\int_{-\infty}^{\infty} W_{x}^{(\mp)}\left(z-z^{\prime}\right) \rho^{( \pm)}\left(z^{\prime}\right) d z^{\prime} x^{(\mp)}(z) .
\end{aligned}
$$

where $\rho_{x}(z)=\rho(z) \cdot x(z)$ is the dipole moment of bunch, and $W_{x}^{(\mp)}(z)$ is the cross-wake function $[7,8]$ for $e^{\mp}$ beams induced by beam-beam interaction, and can be expressed for a flat beam as,

$$
W_{x}^{(\mp)}(z)=\frac{N_{0}^{( \pm)} r_{e}}{\gamma^{(\mp)} \bar{\sigma}_{x}^{2}} W_{N}(\zeta),
$$

where $\zeta=\theta_{P} z / \sigma_{z}, \theta_{P}$ is the Piwinski angle, $\bar{\sigma}_{x}=\left(\sigma_{x}^{+}+\right.$ $\left.\sigma_{x}^{-}\right) / 2$ is the average horizontal $\mathrm{rms}$ beam size of two bunches and $W_{N}(\zeta)$ is normalized cross wake force defined by,

$$
W_{N}(\zeta)=-1+\zeta e^{-\zeta^{2} / 4} \int_{0}^{\zeta / 2} e^{u^{2}} d u
$$

Figure 6 shows the normalized cross wake force $W_{N}(\zeta)$. Unlike the ordinary wake force, the cross wake $W_{x}(z)$ has no causal property and is symmetric for the argument $\mathrm{z}$.

The first term of the momentum kick in Eq. (22) is related to the dipole moment of the opposite beam. While the second term is proportional to its own horizontal displacement $x^{(\mp)}(z)$, this term gives a horizontal tune shift. In the following, we first study the effects of the first term, and in Sec. IV C the second tune-shift term would also be considered.

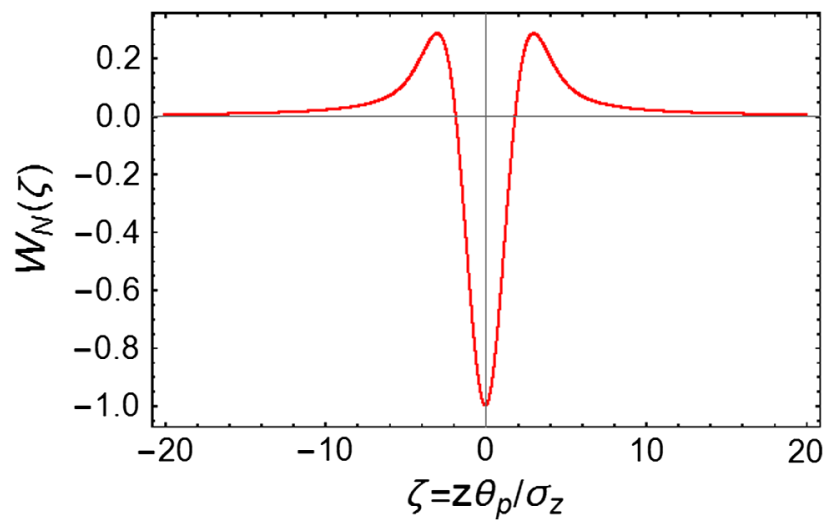

FIG. 6. Normalized cross wake force $W_{N}(\zeta)$, where $\zeta=\theta_{P} z / \sigma_{z}$
The transparency condition is assumed where the electron and positron bunches have the same parameters: $N_{0}^{+} \gamma^{+}=N_{0}^{-} \gamma^{-}, \sigma_{x z}^{+}=\sigma_{x z}^{-}, \nu_{x z}^{+}=\nu_{x z}^{-}$. The stability of colliding bunches is studied separately for the $\sigma$ mode $\rho_{x}^{(+)}(z)=\rho_{x}^{(-)}(z)$ and $\pi$ mode $\rho_{x}^{(+)}(z)=-\rho_{x}^{(-)}(z)$. Representing the dipole moment distribution of two colliding bunches as one same function, the momentum kick is reduced to a normal wake force for a single bunch

$$
\Delta p_{x}(z)=\mp \int_{-\infty}^{\infty} W_{x}\left(z-z^{\prime}\right) \rho_{x}\left(z^{\prime}\right) d z
$$

where the - and + signs represent $\sigma$ and $\pi$ modes, respectively.

\section{B. Transverse mode coupling theory with longitudinal impedance}

We use the normalized coordinates, where $x$ and $p_{x}$ are normalized by

$$
x / \sqrt{\beta_{x}} \rightarrow x, \quad p_{x} \sqrt{\beta_{x}} \rightarrow p_{x} .
$$

Since the dipole amplitudes $x(J, \phi), p_{x}(J, \phi)$ are periodic functions of $\phi$ with period $2 \pi$ in the longitudinal phase space, we expand them as Fourier series,

$x(J, \phi)=\sum_{l=-\infty}^{\infty} x_{l}(J) e^{i l \phi}, \quad p_{x}(J, \phi)=\sum_{l=-\infty}^{\infty} p_{l}(J) e^{i l \phi}$.

In the arc section, the synchro-betatron motion for the vector $\left[x_{l}(J), p_{l}(J)\right]$ is described by the matrix,

$$
M_{0}=e^{-2 \pi i l \nu_{s}(J)}\left(\begin{array}{cc}
\cos \mu_{x} & \sin \mu_{x} \\
-\sin \mu_{x} & \cos \mu_{x}
\end{array}\right) .
$$

Note that the synchrotron tune $\nu_{s}(J)$ is a function of $J$.

At IP, the change of dipole moment, which is induced by cross-wake force, can be expressed as:

$$
\Delta p_{x}(J, \phi)=\mp \beta_{x} \int W_{x}\left(z-z^{\prime}\right) x\left(J^{\prime}, \phi^{\prime}\right) \rho\left(z^{\prime}\right) d z^{\prime} .
$$

Using $\rho\left(z^{\prime}\right)=\int \psi\left(J^{\prime}, \phi^{\prime}\right) d \delta^{\prime}$, we can rewrite the equation

$\Delta p_{x}(J, \phi)=\mp \beta_{x} \int W_{x}\left(z-z^{\prime}\right) x\left(J^{\prime}, \phi^{\prime}\right) \psi\left(J^{\prime}\right) d J^{\prime} d \phi^{\prime}$,

where we have used the fact that the stationary phase-space distribution $\psi(J, \phi)$ in Eq. (9) is only a function of $J$, $\psi(J, \phi)=\psi(J)$, and $z$ is regarded as a function of longitudinal phase space coordinates, $z=z(J, \phi)$, $z^{\prime}=z\left(J^{\prime}, \phi^{\prime}\right)$. It is worth pointing out that $\psi(J)$ is the 
distorted beam distribution. Substituting the expansions in Eq. (27) into Eq. (30), we obtain the momentum change for each azimuthal mode

$$
\Delta p_{l}(J)=\mp \frac{\beta_{x}}{2 \pi} \sum_{l^{\prime}} \int d J^{\prime} W_{l l^{\prime}}\left(J, J^{\prime}\right) \psi\left(J^{\prime}\right) x_{l^{\prime}}\left(J^{\prime}\right),
$$

where

$$
W_{l l^{\prime}}\left(J, J^{\prime}\right)=\iint d \phi d \phi^{\prime} e^{-i l \phi+i l^{\prime} \phi^{\prime}} W_{x}\left(z-z^{\prime}\right) .
$$

The mode index $l$ is infinite, and the argument $J$ is continuous in Eq. (31). To obtain a matrix form, we truncate $l$ at $\pm l_{\max }$, and discretize $J$ at $J_{1}, J_{2}, \ldots, J_{n_{J}}$. The Fourier expansions in Eq. (27) becomes

$$
x\left(J_{i}, \phi\right)=\sum_{l=-l_{\max }}^{l_{\max }} x_{l}\left(J_{i}\right) e^{i l \phi}, \quad i=1,2, \ldots, n_{J} .
$$

Here, $n_{J}, l_{\max }$ should be investigated carefully such that the dominant head-tail mode could not be ignored. $p_{x}$ is truncated and discretized in the same way. In the arc section, the transformation of vector $\left(x_{l}\left(J_{i}\right), p_{l}\left(J_{i}\right)\right)$ in Eq. (28) becomes

$$
M_{0}=e^{-2 \pi i l_{s}\left(J_{i}\right)}\left(\begin{array}{cc}
\cos \mu_{x} & \sin \mu_{x} \\
-\sin \mu_{x} & \cos \mu_{x}
\end{array}\right) .
$$

where the dimension of the matrix $M_{0}$ is $\left(2\left(2 l_{\max }+1\right) \times\right.$ $\left.n_{J}\right)^{2}$. The momentum kick of Eq. (31) is also converted to

$$
\begin{aligned}
\Delta p_{l}\left(J_{i}\right) & =\mp \frac{\beta_{x}}{2 \pi} \sum_{l^{\prime}} \sum_{i^{\prime}} \Delta J_{i^{\prime}} W_{l l^{\prime}}\left(J_{i}, J_{i^{\prime}}\right) \psi\left(J_{i^{\prime}}\right) x_{l^{\prime}}\left(J_{i^{\prime}}\right) \\
& \equiv \beta_{x} M_{l i l^{\prime} i^{\prime}} x_{l^{\prime}}\left(J_{i^{\prime}}\right) .
\end{aligned}
$$

The transformation at IP, therefore, can be written in a condensed matrix form,

$$
M_{W}=\left(\begin{array}{cc}
1 & 0 \\
\beta_{x} M_{l i l^{\prime} i^{\prime}} & 1
\end{array}\right) .
$$

We have obtained the one-to-one correspondence between $(z, \delta)$ and $(J, \phi)$ in Sec. II B. The integration for $\phi$ and $\phi^{\prime}$ in Eq. (32) could be rewritten as a summation, therefore,

$$
\begin{aligned}
W_{l l^{\prime}}\left(J_{i}, J_{i^{\prime}}\right)= & \sum_{j} \sum_{j^{\prime}} e^{-i l \phi_{j}+i l^{\prime} \phi_{j^{\prime}}} W_{x}\left(z\left(J_{i}, \phi_{j}\right)\right. \\
& \left.-z\left(J_{i^{\prime}}, \phi_{j^{\prime}}\right)\right) \Delta \phi_{j} \Delta \phi_{j^{\prime}} .
\end{aligned}
$$

Finally, the stability of the colliding beams is determined by the eigenvalues $\left(\lambda^{\prime} s\right)$ of the revolution matrix $M_{0} M_{W}$.

\section{ANALYSIS OF COUPLING EFFECTS}

In this section, our action discretization method and the conventional radial mode expansion method will be used to study the beam-beam instability without the influence of longitudinal impedance. This could be a cross-check for our formalism. Next, we consider the effects of impedance based on our formalism. Finally, we present the results where the beam-beam tune shift term is also included.

\section{A. Eigensystem without longitudinal impedance: Cross-check for the formalism}

In Sec. III we have introduced our method. To check our formalism, we neglect the influence of impedance. Without the impedance, the solution to the Vlasov equation in Eq. (8) is

$$
\psi(J)=\frac{1}{2 \pi \varepsilon_{z}} \exp \left(-J / \varepsilon_{z}\right),
$$

where $J$ can be expressed as $J=\left(z^{2} / \beta_{z}+\beta_{z} \delta^{2}\right) / 2$, $\epsilon_{z}=\sigma_{z} \sigma_{\delta}$. The radial mode expansion method and action discretization method are both used to study the stability of colliding bunches.

First, we follow the approach [8] to consider the radial mode expansion for the Eq. (27). Using the orthogonality of Laguerre polynomials, each azimuthal mode is splitting into a family of radial modes,

$$
x_{l}(J)=\sum_{k=0}^{\infty} x_{k l} \sqrt{\frac{k !}{(|l|+k) !}}^{|l| / 2} L_{k}^{(|l|)}(\hat{J}),
$$

where $\hat{J}=J / \epsilon_{z}$. Similar to Eq. (33), we truncate $k$ at $k_{\max }$. $p_{l}(J)$ is also expanded in the same manner. Since the longitudinal phase space is elliptical, $W_{l l^{\prime}}\left(J, J^{\prime}\right)$ in Eq. (32) can be converted into an impedance form,

$$
\begin{aligned}
W_{l l^{\prime}}\left(J, J^{\prime}\right) & =\iint d \phi d \phi^{\prime} e^{-i l \phi+i l^{\prime} \phi^{\prime}} W_{x}\left(z-z^{\prime}\right) \\
& \approx 2 \pi i^{l-l^{\prime}-1} \int_{-\infty}^{\infty} d \omega Z_{\perp}(\omega) J_{l}\left(\frac{\omega r_{z}}{c}\right) J_{l^{\prime}}\left(\frac{\omega r_{z}^{\prime}}{c}\right),
\end{aligned}
$$

where $r_{z}=\sqrt{2 \beta_{z} J}, z=r_{z} \cos \phi$. The property of the Bessel function has been used

$$
\frac{1}{2 \pi} \int_{0}^{2 \pi} d \phi e^{i l \phi-i x \cos \phi}=i^{-l} J_{l}(x) .
$$

$Z_{\perp}(\omega)$ is the Fourier transform of the cross-wake $W_{x}(z)$,

$$
Z_{\perp}(\omega)=i \int_{-\infty}^{\infty} W_{x}(z) e^{-i \omega z / c} \frac{d z}{c} .
$$


Since $W_{x}(z)$ is symmetric for the argument $z, Z_{\perp}(\omega)$ is a purely inductive even function of $\omega$. Substituting Eq. (39) into Eq. (31), and using the impedance expression of $W_{l l^{\prime}}\left(J, J^{\prime}\right)$ in Eq. (40), the momentum kick for each mode $\Delta p_{k l}$ then becomes [8]

$$
\begin{aligned}
\Delta p_{k l} & =-\beta_{x} \sum_{k^{\prime} l^{\prime}} i^{l-l^{\prime}-1} \int d \omega Z_{\perp}(\omega) g_{k l}(\omega) g_{k^{\prime} l^{\prime}}(\omega) x_{k^{\prime} l^{\prime}} \\
& \equiv-\sum_{k^{\prime} l^{\prime}} M_{k l k^{\prime} l^{\prime}} x_{k^{\prime} l^{\prime}},
\end{aligned}
$$

where

$$
g_{k l}=\frac{1}{\sqrt{2 \pi k !(|l|+k) !}}\left(\frac{\omega \sigma_{z}}{\sqrt{2} c}\right)^{|l|+2 k} e^{-\omega^{2} \sigma_{z}^{2} / 2 c^{2}} .
$$

Second, the discretization of $J$ in our formalism is taken as follows. We consider particles within $\pm 3 \sigma_{z}$ regions which corresponds $J$ from 0 to $J_{\max } \approx 4.5 \epsilon_{z} . J$ is discretized as $\sqrt{J_{i}}=i \Delta J, i=1 \ldots n_{J} . \Delta J$ is the spacing of the grid. In this paper, we take $n_{J}=40, l_{\max }=8$ in the discretization method, and $k_{\max }=80, l_{\max }=8$ in the radial mode expansion method.
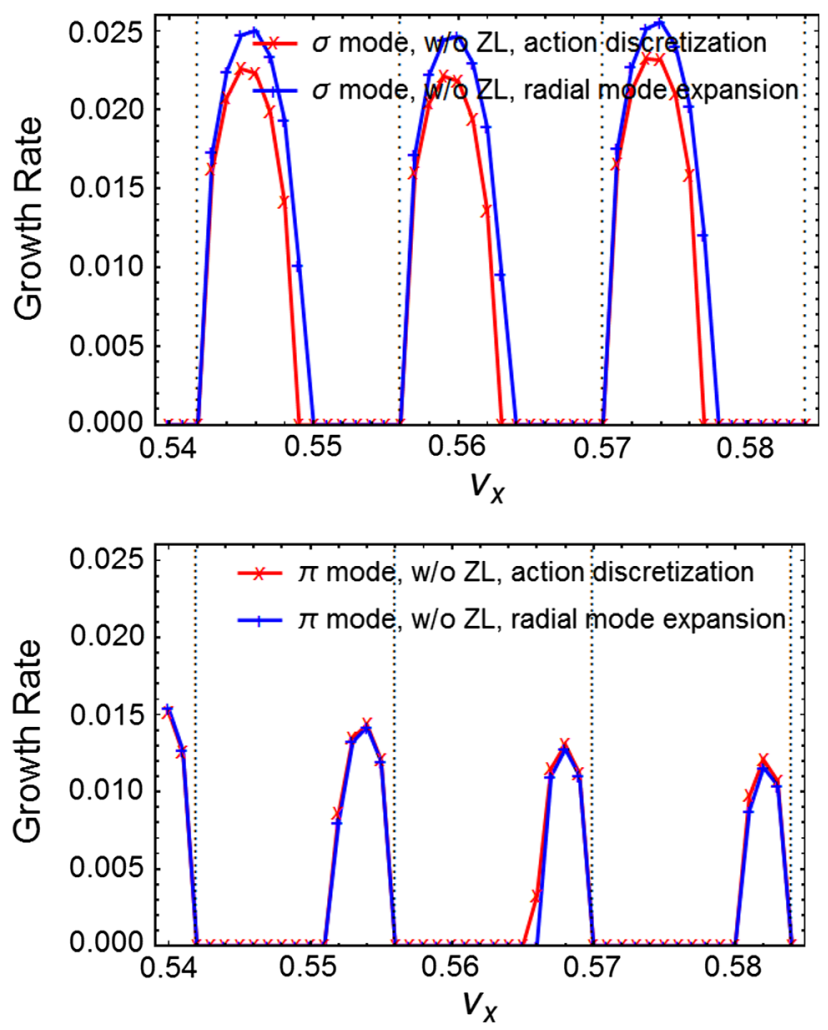

FIG. 7. Growth rate vs horizontal tune without longitudinal impedance (ZL). The upper and lower plots show the results of $\sigma$ mode and $\pi$ mode, respectively. The vertical lines are synchrobetatron sidebands $\nu_{x}=0.5+n \nu_{s}, \nu_{s}=0.014$.
Figure 7 shows the comparison of the two methods. The growth rate is defined as the largest $\log |\lambda|$. One can see that the growth rates computed by the two methods are not too much different from each other. Actually, if we reduce the spacing $\Delta J$ (increase $n_{J}$ ) and increase the truncation order $l_{\max }, k_{\max }$, the difference can be reduced. For example, in action discretization method, the growth rate almost converges when $n_{J}=80$. When $n_{J}$ increases from 40 to 80 , the value of growth rate at $\nu_{x}=0.574$ increases by about $5 \%$. This increment slightly reduces the difference between the two method, but the computing time becomes four times. Its a balance between efficiency and accuracy. One can see that the peaks do not coincide with the synchro-betatron resonance lines. This could be explained by the absence of the tune-shift term in Eq. (22). The unstable regions are above/below the resonance lines for $\sigma / \pi$ mode, respectively. This indicates that the tune shift induced by the cross-wake force is negative for the $\sigma$ mode and positive for the $\pi$ mode since it is generally the most unstable near the resonance lines.

We refer to $\nu=\tan ^{-1}(\operatorname{Im} \lambda / \operatorname{Re} \lambda) /(2 \pi)$ as the eigentune. The eigentune as a function of bunch population $N / N_{0}$ is shown in Fig. 8 where $\nu_{x}=0.546, \nu_{s}=0.014$. For $\nu<0.5$, it is wrapped and $\bar{\nu}=1-\nu$ is shown in the plot. The lines start from $\nu=\nu_{x}+l \nu_{s}$ for $l>-4$ and $\bar{\nu}=1-$ $\left(\nu_{x}+l \nu_{s}\right)$ for $l \leq-4$ at $N=0$. One can see that the eigentunes shift downward for $\sigma$ mode and upward for $\pi$ mode. The $\nu=0.504$ mode $(l=-3)$ shifts downward, approaching 0.5 and an instability occurs at $N \approx 0.6 N_{0}$,

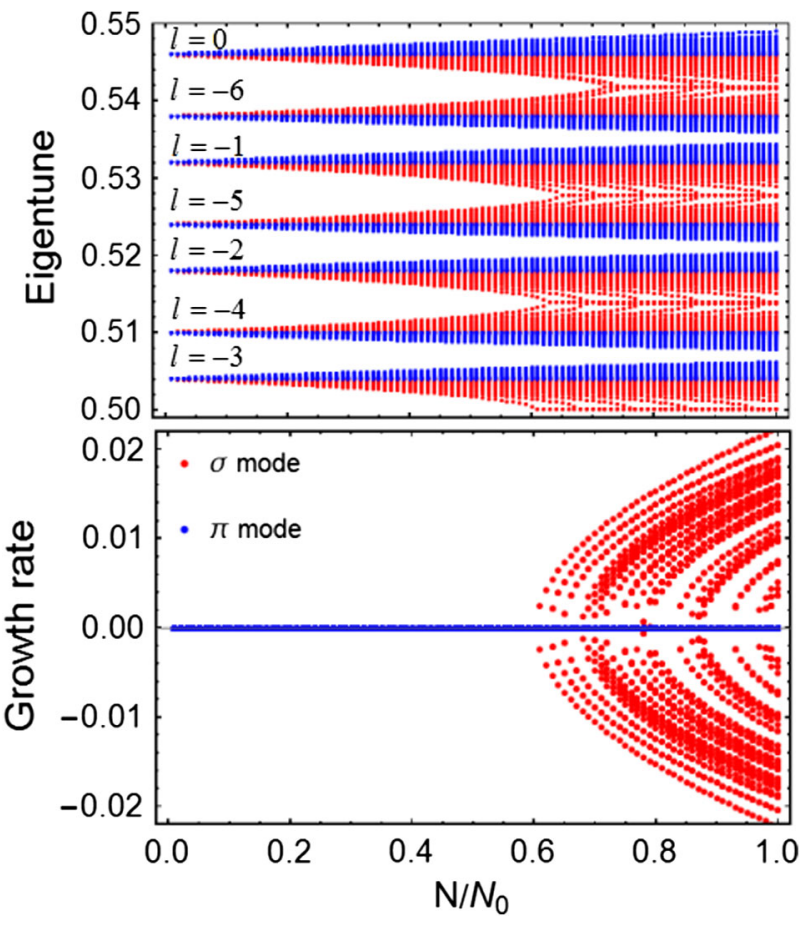

FIG. 8. Eigenvalues of $\sigma$ and $\pi$ modes as a function of bunch population without longitudinal impedance, where $\nu_{x}=0.546$. The red and blue points represent the $\sigma$ and $\pi$ modes, respectively. 


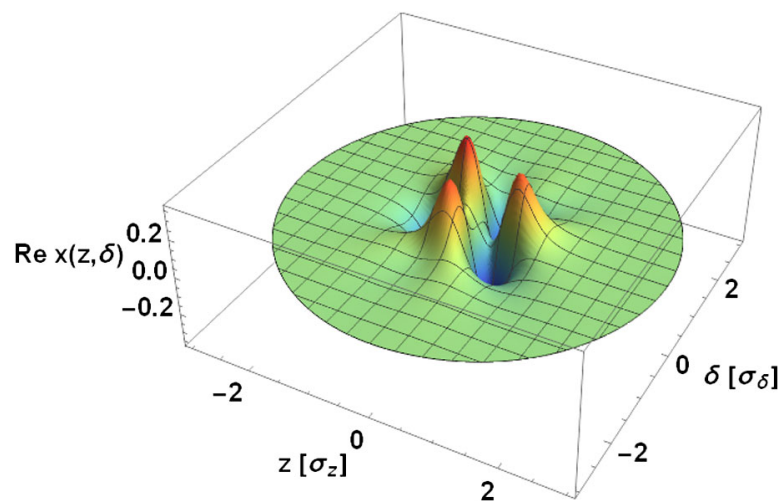

FIG. 9. Real part of dipole amplitude $x(z, \delta)$ as a function of $z$ and $\delta$ associated to the mode with fastest growth rate for $\sigma$ mode, where $\nu_{x}=0.546$. The $z$ and $\delta$ axes are in units of $\sigma_{z}$ and $\sigma_{\delta}$, respectively. $x$ is in arbitrary unit.

which causes the half-integer resonance. This mode has the fastest growth rate. We note that mode coupling occurs between only modes with the same parity. For example, the $l=-2,-4$, and $l=-1,-5$ modes merge and an imaginary part of eigentune appears at $N>0.6 N_{0}$.

Using the eigenvector, we can reconstruct the dipole amplitude $x(J, \phi)$ in Eq. (27). Figure 9 presents the real part of dipole amplitude $x(z, \delta)$ of the most unstable mode as a function of $z$ and $\delta$ at the design population $N=N_{0}$. There are three peaks along the azimuthal which means the $l= \pm 3$ modes dominate. This is consistent with the result shown in Fig. 8 where the $l=-3$ mode is dominant and has the fastest growth rate.

Figure 10 presents the real part of eigenvector $x_{l}(J)$ of the most unstable mode as a function of $J$ at the design population $N=N_{0}$. As one can see from the figure that the excited modes exist only for $l= \pm 1, \pm 3, \pm 5 \ldots$ and no for $l=0, \pm 2, \pm 4 \ldots$. That is to say, the most unstable

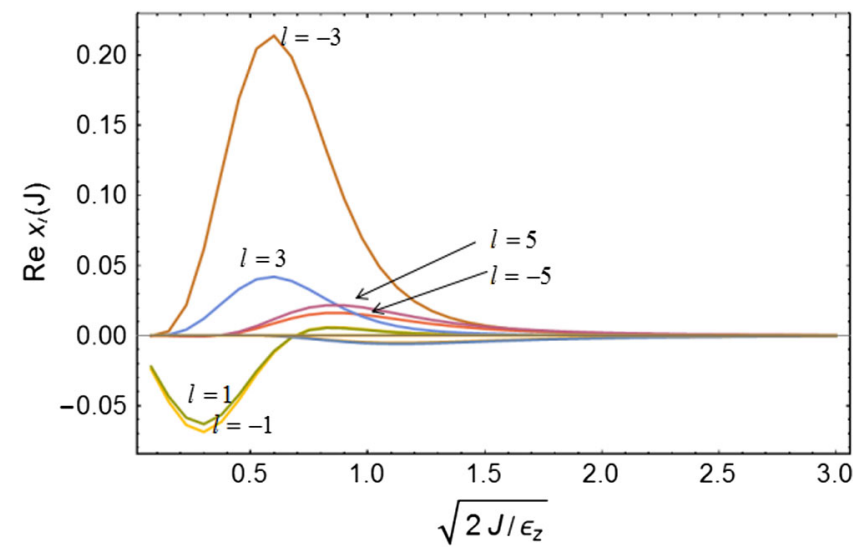

FIG. 10. Real part of eigenvector $x_{l}(J)$ of the most unstable mode as a function of $J$ for $\sigma$ mode, where $\nu_{x}=0.546, N=N_{0}$. $x_{l}(J)$ is calculated with linear interpolation. The $J$ axes is in the unit of $\sqrt{2 J / \epsilon_{z}}$. eigenmode is induced by the coupling of modes with the same parity, and there is no mode mixing with different parity modes when the longitudinal impedance is not considered. The following two mechanisms could explain: (1) the cross-wake impedance $Z_{\perp}(\omega)$ is a purely inductive even function of $\omega$, and (2) the longitudinal phase space trajectory is elliptical, so we have the impedance form of Eq. (40). The interaction between two modes is given by the overlap integral of the two-mode spectra with impedance. Therefore, the matrix element $M_{k l k^{\prime} l^{\prime}}$ in Eq. (43) are 0 for $l, l^{\prime}$ with different parities and retained for the exchange of $l \leftrightarrow l^{\prime}, k \leftrightarrow k^{\prime}$ for $l, l^{\prime}$ with same parity. That is, the interaction occurs only in modes with the same parity. We will see that these coupling features are quite different when the longitudinal impedance is considered.

\section{B. Eigensystem with longitudinal impedance}

When we consider the longitudinal impedance, the longitudinal phase space trajectory is distorted. Equation (40) (43) are not valid, and we have to resort to the discrete expansions of Eq. (33) (35) and numerical evaluation of Eq. (37). In the following parts, we study the coupling effects of beam-beam interaction and longitudinal impedance based on our new formalism.
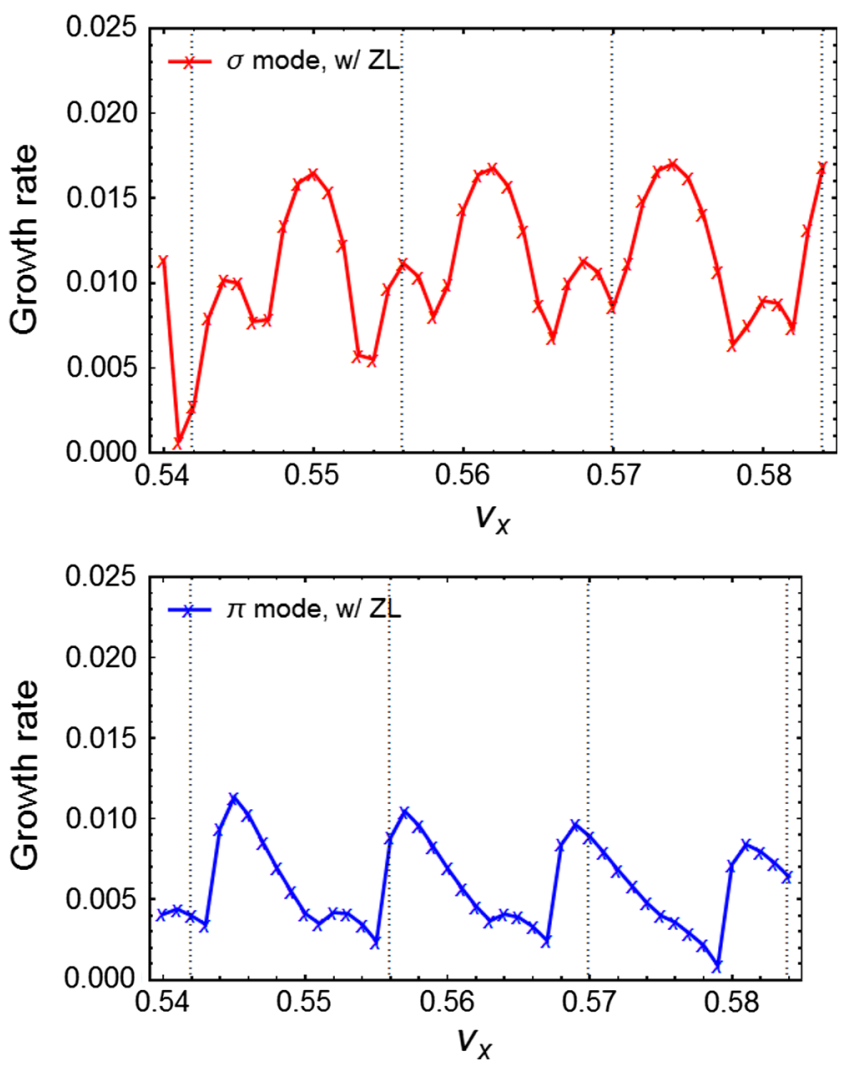

FIG. 11. Growth rate vs horizontal tune with longitudinal impedance (ZL). The red and blue points represent the $\sigma$ and $\pi$ modes, respectively. 
Figure 11 shows the growth rate for various horizontal tunes where the longitudinal impedance is included. Comparing to the results without impedance shown in Fig. 7, the gap $\Delta \nu$ between two neighboring peaks is reduced from 0.014 to 0.011 . Note that $\nu_{s}(J) \approx 0.011$ is the synchrotron tune of small amplitude particle. It indicates that the instability mainly comes from small amplitude particle. This is understandable since the momentum change of each mode in Eq. (35) is proportional to the particle density $\psi(J)$. Besides the change of gap $\Delta \nu$, the once-stable working tune has turned unstable for both $\sigma$ and $\pi$ modes when we consider the influence of impedance. We will see that these changes result from the destruction of symmetry of the longitudinal phase space trajectory and the incoherent synchrotron tune shift, which are shown in Fig. 3 and Fig. 4, respectively.

The eigentune spectrum as a function of bunch population for $\sigma / \pi$ modes is shown in Fig. 12, where $\nu_{x}=0.546$. In the plots, the lines start from $\nu=\nu_{x}+l \nu_{s}$. Since the incoherent synchrotron tune shift shown in Fig. 4 is negative, the induced shift of each eigentune is positive for $l<0$. Remember that the tune shift of the $\pi$ mode induced by cross wake is also positive; thus, the total tune shift would be strengthened comparing to that of $\pi$ mode shown in Fig. 8. As a consequence, the modes with different parities are merged at around $N=0.25 N_{0}$. According to Eq. (32), due to the distortion of longitudinal phase space trajectory, different parity modes can interact, and instability starts to appear. By the way, if the trajectory is not distorted, the instability would not appear. More details will be shown and discussed later. As one continues increasing the bunch intensity, modes with the same parity would also couple. A similar analysis has also been done

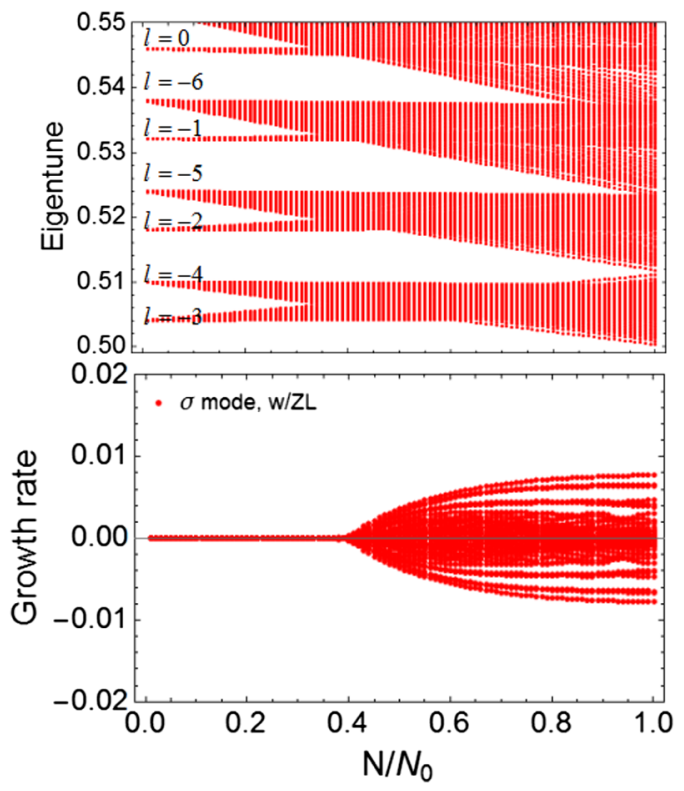

for $\sigma$ mode in Fig. 12. But in this $\sigma$ mode, the tune shift induced by cross wake is negative. Note that the tune shift caused by incoherent synchrotron shift is positive for $l<0$. Therefore, the total tune shift of $\sigma$ mode is smaller than that of $\pi$ mode, which explains that there is only one instability threshold in the $\sigma$ plot.

The following two simplification cases are studied to investigate the effects of the incoherent synchrotron tune shift and the distortion of longitudinal phase space trajectory, separately: 1 . The trajectory is elliptical and we consider the synchrotron tune shift; 2 . we consider the distortion of elliptical trajectory but keep the synchrotron tune constantly $\nu_{s}(J)=0.014$. These assumptions may not be physical, but they help us understand the mechanism of the instability.

Figure 13 shows the comparison of growth rate between case 1 and case without longitudinal impedance for $\sigma / \pi$ modes. As one can see from the figure, the gap between two neighboring peaks is reduced to $\Delta \nu \approx 0.011$. There are no structures between the peaks, and the width of stable area is squeezed seriously both for $\sigma$ and $\pi$ modes, especially at large $\nu_{x}$. Figure 14 shows the variation in the eigentune and growth rate of $\sigma / \pi$ modes, where $\nu_{x}=0.546$. One can see from the figure that although different parity modes are merged at around $N=0.25 N_{0}$, the instability does not occur. We can conclude that if the longitudinal phase trajectory is not distorted, different parity modes would not be coupled to induce instability.

Figure 15 shows the comparison of growth rate between case 2 and case without longitudinal impedance for $\sigma / \pi$ modes. Since we have assumed a constant synchrotron tune $\nu_{s}(J)=0.014$, the gap between two neighboring peaks is unchanged $\Delta \nu=0.014$. As one can see from the figure, new unstable tune areas appear in the regions where are

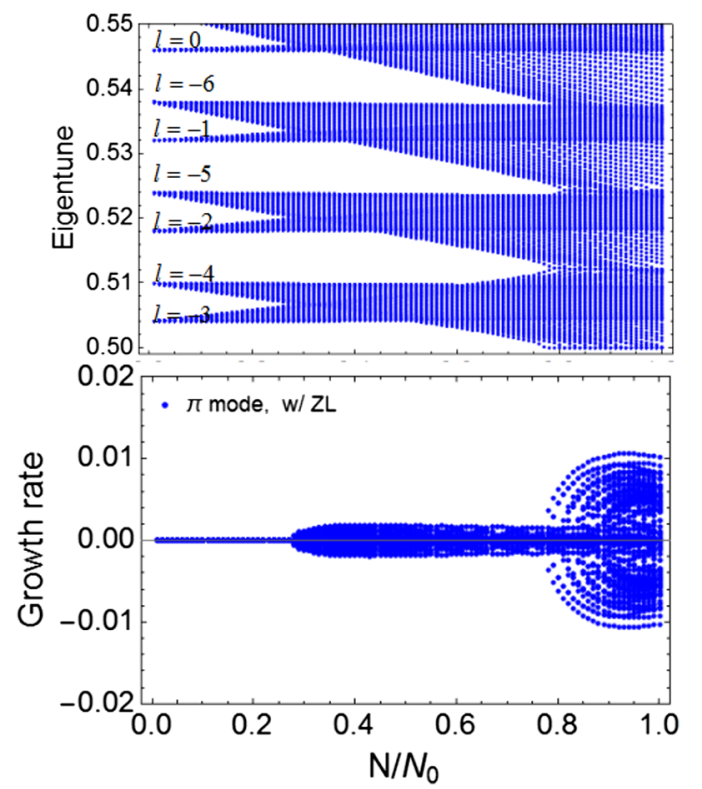

FIG. 12. Eigenvalues of the $\sigma$ mode (left) and $\pi$ mode (right) as a function of bunch population with longitudinal impedance (ZL), where $\nu_{x}=0.546$. 

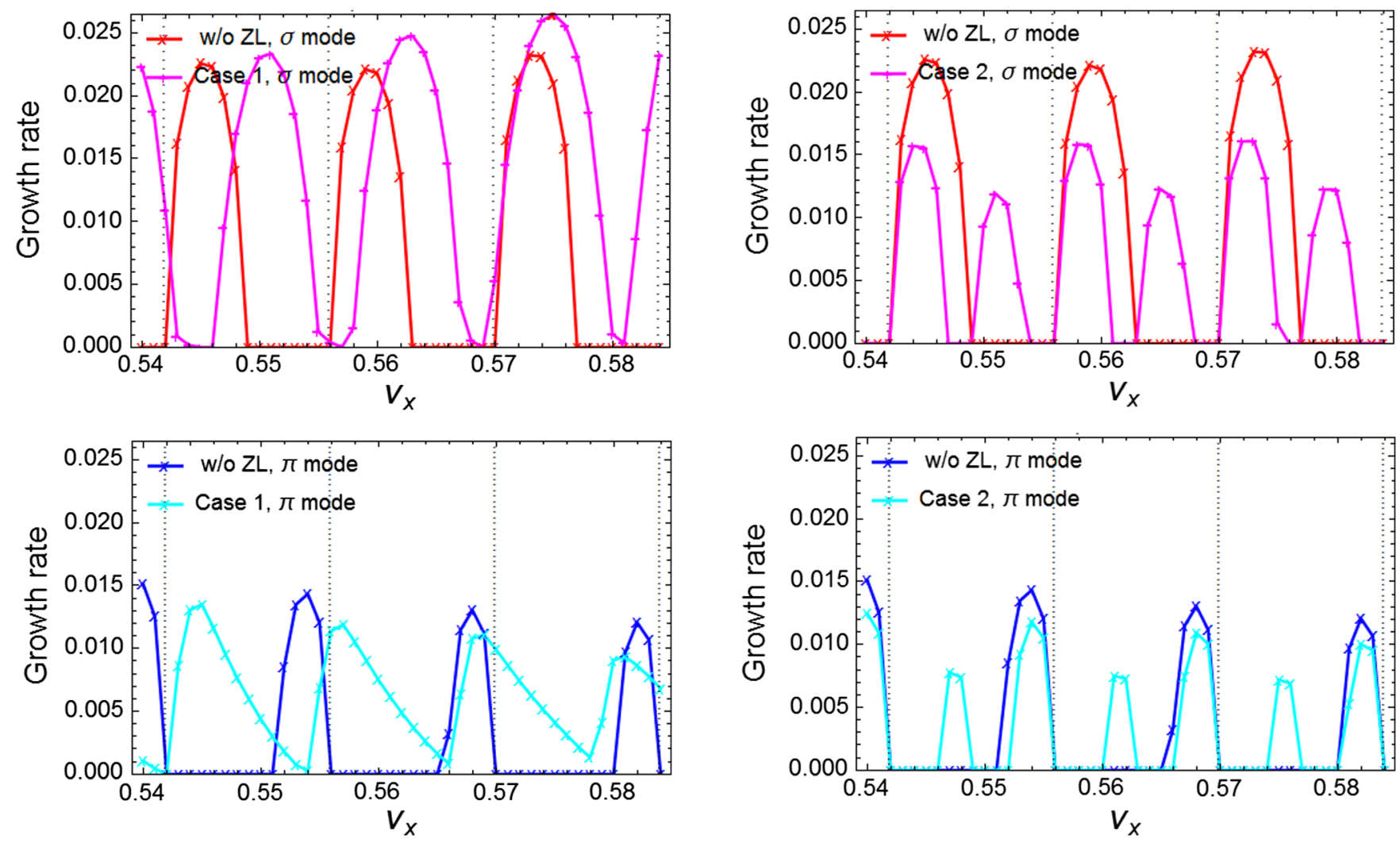

FIG. 13. Comparison of growth rate between case 1 and case without longitudinal impedance for $\sigma / \pi$ modes. In case 1 , the trajectory is elliptical and we consider the incoherent synchrotron tune shift.

FIG. 15. Comparison of growth rate between case 2 and case without longitudinal impedance for $\sigma / \pi$ modes. In case 2 , we consider the distortion of elliptical trajectory but keep the synchrotron tune constantly $\nu_{s}(J)=0.014$.
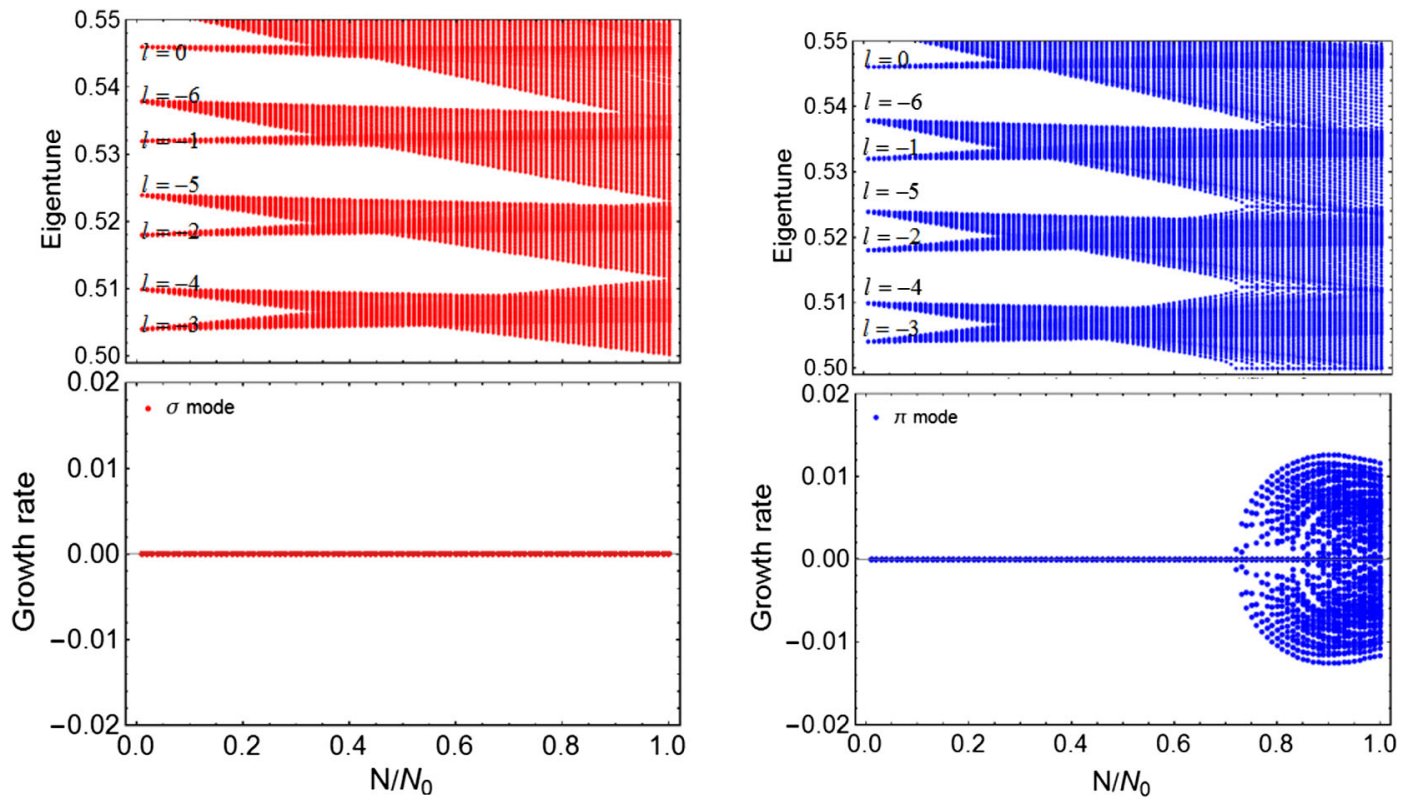

FIG. 14. Eigenvalues of $\sigma$ mode (left) and $\pi$ mode (right) as a function of bunch population, where $\nu_{x}=0.546$. In this case, we assume no distortion of elliptical trajectory but keep the incoherent synchrotron tune shift. 


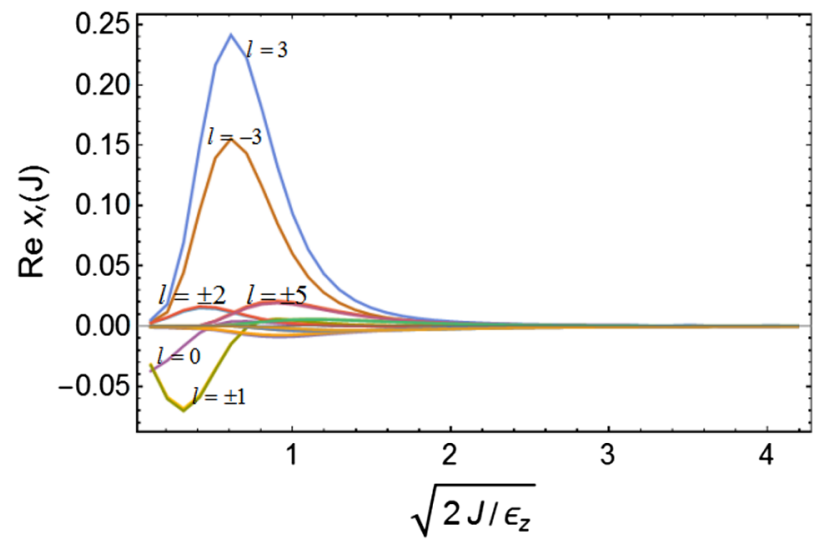

FIG. 16. Real part of eigenvector $x_{l}(J)$ of the most unstable mode as a function of $J$ for $\sigma$ mode, where $\nu_{x}=0.546, N=N_{0}$. $x_{l}(J)$ is calculated with linear interpolation. In this case we consider the distortion of longitudinal phase space trajectory but keep the synchrotron tune constantly.

stable in the case without impedance. These new unstable tunes can be explained by the breaking of longitudinal phase space symmetry. Since the cross-wake $W_{x}(z)$ is symmetric for the argument $z, W_{l l^{\prime}}\left(J, J^{\prime}\right)$ in Eq. (32) vanishes for $l, l^{\prime}$ with different parities if the phase space is elliptical. However, $W_{l l^{\prime}}\left(J, J^{\prime}\right)$ is finite if the phase space is not symmetric. It indicates from Eq. (31) that the azimuthal modes would couple with each other. As shown in Fig. 10, without considering the effects of impedance, only the same parity modes would couple with each other. However, with the distortion of trajectory, as shown in Fig. 16, all parity modes $l=0, \pm 1, \pm 2, \pm 3 \ldots$ are excited and could be mixing.

To sum up, when the longitudinal impedance is included, the longitudinal phase space trajectory would be distorted. The distortion is the condition that different parity modes couple to instability. By the way, the instability growth rate is slightly reduced compared to the case without longitudinal impedance.

\section{Eigensystem with beam-beam tune shift}

In the previous sections, we only study the first term of cross wake force given in Eq. (22). In this subsection, we would also consider the effects of the second term. We will see that the second term only gives rise to a shift of horizontal working tune.

Just like what we've done for Eq. (31), the dipole moment kick is expanded into azimuthal modes

$$
\begin{aligned}
\Delta p_{l}(J)= & \mp \frac{1}{2 \pi} \sum_{l^{\prime}} \int W_{l l^{\prime}}\left(J, J^{\prime}\right) \psi\left(J^{\prime}\right) x_{l^{\prime}}\left(J^{\prime}\right) d J^{\prime} \\
& +\frac{1}{2 \pi} \sum_{l^{\prime}}\left\{\int \bar{W}_{l l^{\prime}}\left(J, J^{\prime}\right) \psi\left(J^{\prime}\right) d J^{\prime}\right\} \cdot x_{l^{\prime}}(J),
\end{aligned}
$$

where

$$
\begin{aligned}
& W_{l l^{\prime}}\left(J, J^{\prime}\right)=\iint d \phi d \phi^{\prime} e^{-i l \phi+i l^{\prime} \phi^{\prime}} W\left(z-z^{\prime}\right) \\
& \bar{W}_{l l^{\prime}}\left(J, J^{\prime}\right)=\iint d \phi d \phi^{\prime} e^{-i\left(l-l^{\prime}\right) \phi} W\left(z-z^{\prime}\right) .
\end{aligned}
$$

The second tune-shift term, proportional to each azimuthal amplitude $x_{l^{\prime}}(J)$, is the same both in $\sigma$ and $\pi$ modes. Following the procedure laid out in Sec. III B, we can obtain the eigenvalues of the revolution matrix $M_{0} M_{W}$.

Figure 17 shows the growth rate for various horizontal tunes where we consider the second tune-shift term but ignore the longitudinal impedance. As one can see from the figure, when $\nu_{x}=0.5+n \nu_{s}$, the collision is most unstable for the $\sigma$ mode. This may be explained as follows: We have shown before that the tune shift induced by the cross-wake force (first term) is negative for $\sigma$ mode $\left(\Delta \nu_{c w: \sigma}<0\right)$; For an $e^{+} e^{-}$collider, the coherent beam-beam tune shift (second term) is positive $\left(\Delta \nu_{b b}>0\right)$. Thus, the tune shift induced by the two terms cancel each other out in the $\sigma$ mode. As for the $\pi$ mode, since the tune shift induced by the two terms are both positive, the total tune shift would be strengthened.

Figure 18 shows the growth rate for various horizontal tunes where we consider both the second tune-shift term
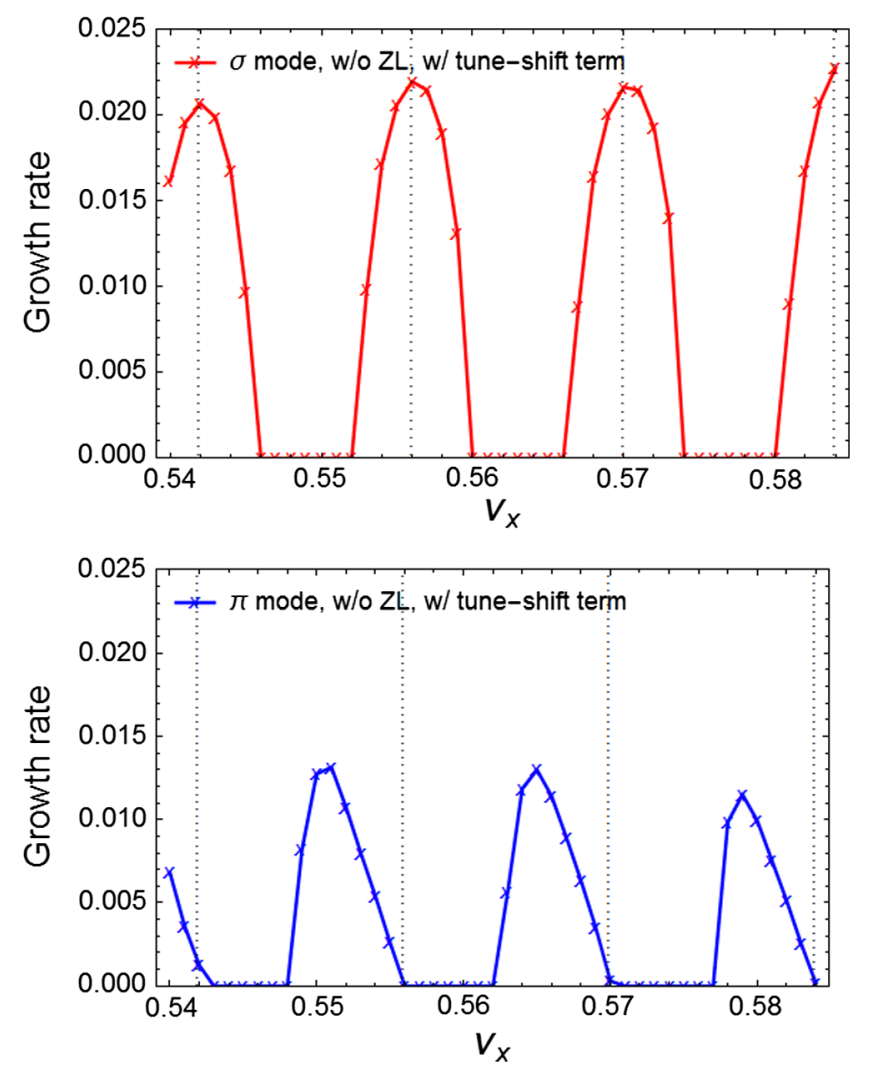

FIG. 17. Growth rate vs horizontal tune, where we also consider the second tune-shift term of Eq. (22) but without the longitudinal impedance (ZL). The red and blue points represent the $\sigma$ and $\pi$ modes, respectively. 

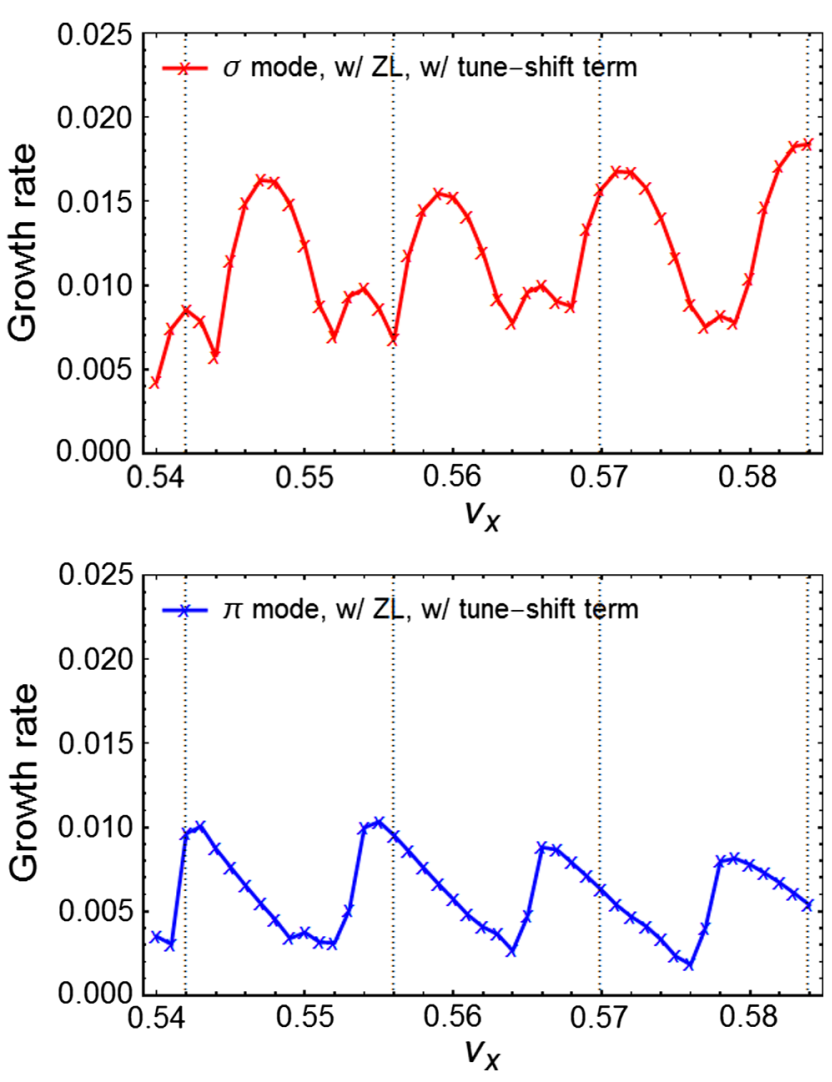

FIG. 18. Growth rate vs horizontal tune, where we also consider the second tune-shift term of Eq. (22) and longitudinal impedance (ZL). The red and blue points represent the $\sigma$ and $\pi$ modes, respectively.

and the longitudinal impedance. Comparing to Fig. 11, it is clear that the second term gives rise to a horizontal tune shift. Due to the incoherent synchrotron tune shift induced by impedance, the amount of the tune shift is different from the case without impedance.

In short, the second tune-shift term has little effect on the growth rate and mainly gives rise to the shift of horizontal tune.

\section{CONCLUSIONS}

The beam-beam coherent head-tail instability in collision with a large crossing angle is strongly dependent on the longitudinal beam dynamics. The longitudinal impedance would distort the longitudinal phase space trajectory and introduce an incoherent synchrotron tune shift. Moreover, the beam-beam cross wake force is very localized.

In this paper, we have developed a transverse mode coupling analysis method to study the coherent beam-beam instability under the influence of longitudinal impedance. The new approach has been cross-checked with the conventional radial mode expansion method in the case without longitudinal impedance. This method could be used to study transverse instability induced by general wakefields in presence of potential well distortion.
There exist stable horizontal working tune areas separated by $\nu_{s}$ when the longitudinal impedance is not considered. Mode coupling occurs only between azimuthal modes with same parity. The eigenmode is a linear mixture of unperturbed modes of same parity. This coupling feature is derived from the symmetry of the cross-wake function $W_{x}(z)$ and the elliptical trajectory of the particle in longitudinal phase space.

The longitudinal impedance would distort the trajectory and produce the incoherent tune shift. It is found that the stable working tune area is almost squeezed out when we consider the impedance. The distance between two neighboring most unstable horizontal tunes is reduced from 0.014 (unperturbed $\nu_{s}$ ) to 0.011 . It agrees with the incoherent synchrotron tune shift for low synchrotron amplitude particle. Mode coupling behavior is quite different from that of without impedance. Modes with different parities could be coupled with each other. The eigenmode now is a linear mixture of unperturbed modes of odd and even parities, which is the consequence of symmetry breaking of the longitudinal phase space trajectory.

Several aspects of this work can be studied in the future. The strong-strong simulation shows that in future high energy $e^{+} e^{-}$collider, the beamstrahlung effect plays a vital role in beam-beam interaction [9]. It is interesting to develop an analytical method for the combined effects of beamstrahlung and longitudinal impedance. It is also natural to study the mode coupling theory for beam-beam interaction under the effects of both transverse and longitudinal impedance.

\section{ACKNOWLEDGMENTS}

This work is supported by National Key Programme for S\&T Research and Development, China (Grant No. 2016YFA0400400), National Natural Science Foundation of China (No. 11775238) and Chinese Academy of Sciences Visiting Professorship for Senior International Scientists (Grant No. 2019VMA0034).

[1] F. Ruggiero and F. Zimmermann, Luminosity optimization near the beam-beam limit by increasing bunch length or crossing angle, Phys. Rev. ST Accel. Beams 5, 061001 (2002).

[2] D. Shatilov, E. Levichev, E. Simonov, and M. Zobov, Application of frequency map analysis to beam-beam effects study in crab waist collision scheme, Phys. Rev. ST Accel. Beams 14, 014001 (2011).

[3] P. Raimondi, Status on SuperB effort, in Proceedings of the 2nd SuperB Workshop (Frascati, 2006).

[4] M. Zobov et.al, Test of "Crab-Waist" Collisions at the DAФNE $\Phi$ Factory, Phys. Rev. Lett. 104, 174801 (2010). 
[5] K. Ohmi, Beambeam and electron cloud effects in cepc/ fcc-ee, Int. J. Mod. Phys. A 31, 1644014 (2016).

[6] K. Ohmi, Simulated Beam-beam Limits for Circular Lepton and Hadron Colliders, in 7th International Particle Accelerator Conference (2016) p. MOZA01.

[7] K. Ohmi, N. Kuroo, K. Oide, D. Zhou, and F. Zimmermann, Coherent Beam-Beam Instability in Collisions with a Large Crossing Angle, Phys. Rev. Lett. 119, 134801 (2017).

[8] N. Kuroo, K. Ohmi, K. Oide, D. Zhou, and F. Zimmermann, Cross-wake force and correlated head-tail instability in beam-beam collisions with a large crossing angle, Phys. Rev. Accel. Beams 21, 031002 (2018).

[9] Y. Zhang, N. Wang, C. Lin, D. Wang, C. Yu, K. Ohmi, and M. Zobov, Self-consistent simulations of beam-beam interaction in future $e^{+} e^{-}$circular colliders including beamstrahlung and longitudinal coupling impedance, Phys. Rev. Accel. Beams 23, 104402 (2020).

[10] A.Chao, Physics of Collective Beam Instabilities in High Energy Accelerators (Wiley-Interscience Publication, New York, 1993) and references therein.
[11] F. Ruggiero, Transverse mode coupling instability due to localized structure, Part. Accel. 21, 49 (1986).

[12] K.Oide, a mechanicsm of longitudinal single bunch instability in storage rings, Part. Accel. 51, 43 (1995).

[13] Y. Cai, Linear theory of microwave instability in electron storage rings, Phys. Rev. ST Accel. Beams 14, 061002 (2011).

[14] N. Wang and et al., Mitigation of coherent beam instabilities in cepc, presented at ICFA mini-Workshop on MCBI, Zermatt, Switzerland, Sep. 2019.

[15] CEPC Study Group, CEPC Conceptual Design Report: Volume 1-Accelerator (2018), arXiv:1809.00285.

[16] J.Haissinski, Exact longitudinal equilibrium distribution of stored electrons in the presence of self-fields, Nuovo Cimento Soc. Ital. Fis. 18B, 72 (1973).

[17] S. Y. Lee, Accelerator Physics, 2nd ed. (World Scientific Publishing, Singapore, 2004).

[18] K. Hirata, Analysis of Beam-Beam Interactions with a Large Crossing Angle, Phys. Rev. Lett. 74, 2228 (1995). 